\title{
A Wireless and Context-Aware ECG Monitor : An iMote2 Based Portable System
}

\author{
F Spadini $^{1}$, F Vergari ${ }^{2}$, L Nachman ${ }^{3}$, C Lamberti $^{2}$, T Salmon Cinotti ${ }^{1}$ \\ ${ }^{1}$ ARCES - University of Bologna, Bologna, Italy \\ ${ }^{2}$ University of Bologna, Bologna, Italy \\ ${ }^{3}$ Intel Corporation, USA
}

\begin{abstract}
This paper presents a portable monitoring system for ECG data. The system consists of an iMote 2 with its sensor board (a platform from Intel Research), a custom ECG board, and a Bluetooth module capable of transmitting information to a smart phone. The iMote 2 has the task of contextualizing the ECG data by adding not only accelerometer data, but ambient information as well. The integration of a subject's activity from accelerometers and environmental data like temperature, humidity, and light intensity will give a better general picture of where the changes in ECG data come from. The system visualizes the information on a portable Windows Mobile handheld with the possibility of transmitting the information to a remote server for later use or re-use. The overall result is a system which provides a clean ECG signal for classification to be run on the iMote2, where further processing and visualization can be performed by add-on mobile devices.
\end{abstract}

\section{Introduction}

In the past, telemedicine has been predominantly focused on gathering patient data and sending it to a remote terminal for processing or interpretation. With the continuing advancements in processor technology and machine learning, systems have progressed from their initial sample and send counterparts to more active systems. Current research $[1,2]$ has been progressively adding "intelligence" to the local device by trying to provide user alarms as well as performing local processing and compression on captured ECG holter data. Thanks to the steady beat of Moore's law, not only have processors become smaller and more powerful, but so have sensor technologies. With the advent of MEMS (Micro Electro-Mechanical Systems) sensors and their required signal conditioners, researchers have started adding contextual information to portable telemedicine systems as well [3]. A great deal of research has gone into the combination of ECG and accelerometer data to provide a more complete understanding of the patient's conditions and the situations they occur in.

In the light of current research, a portable monitoring system is being developed which is in its prototype stages. The system is based on a custom ECG board, an iMote2 [4] from Intel Research, a Bluetooth module and a UMTS enabled smart phone. Unlike its predecessors, the system is designed to provide additional and more diverse kinds of context to the system. The initial design incorporates the sensors offered from the Intel sensorboard, including tri-axial accelerometer, temperature, humidity and light intensity as well as sensors not onboard, such as ECG data. The basis for offering environmental information is that by giving a more complete view of the patient's situation, a private care physician can glean a better understanding of where the variations in ECG data come from.

This paper is organized into the following sections: Section II will cover the overall system, Section III provides an explanation of the context acquisition chain, Section IV provides insight into the ECG module as well the algorithms used, Section IV provides details as to the systems power consumption and other preliminary results, and Section $\mathrm{V}$ concludes this study.

\section{System architecture}

The system architecture can be seen in Figure 1, of which the prototype encompasses the on-person platform. The overall goal is to have viable context information processed on the iMote 2 and then sent to a smart phone in order to be further aggregated and stored in a remote context management infrastructure [5]. From a high level viewpoint, this system allows the re-use of context information gathered from multiple sources. As initial inputs, the sensors on the left of Figure 1 are fed into the iMote 2 for local processing. Local processing is performed in order to abstract the raw sensor data as well as reduce the over- 


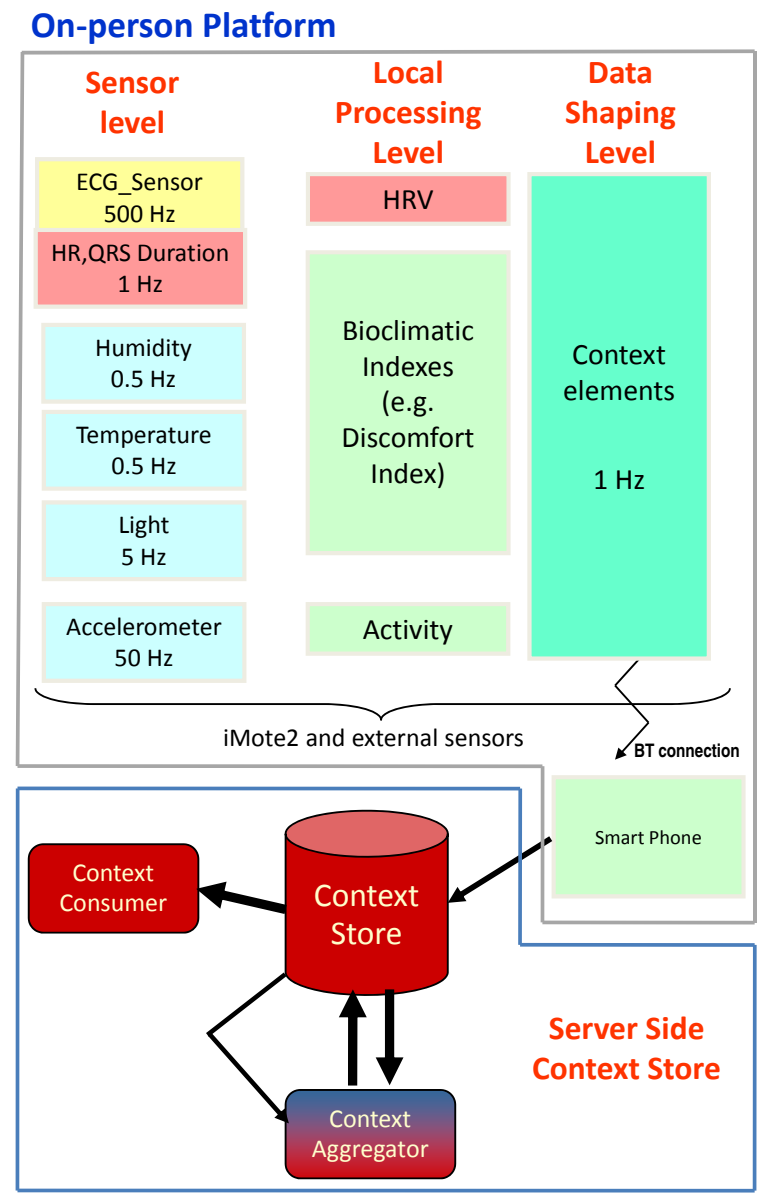

Figure 1. System Overview

all wireless traffic in the system. As shown in other research [6], this will decrease the possibility of congestion on both wireless links in addition to the overall power consumption of the system.

\section{Context acquisition chain}

The context acquisition chain is made up of four primary components:

- iMote2 Platform

- Intel basic sensorboard for iMote2

- Bluetooth adapter carrying a Parani ESD200

- ECG acquisition board

The iMote2 is an advanced wireless sensor node originally developed by Intel Research. The processor board carries a low-power XScale PXA271 as well as an 802.15.4 radio from Chipcon. Currently the data acquisition and processing software is run on top of TinyOS 2, a well known operating system for sensor network research.

Sensor data is provided by a daughter board created in the Intel research labs known as the basic sensor- board. This device carries an ST Micro LIS3L02DQ 3d accelerometer, Sensirion SHT15 temperature/humidity sensor, TAOS TSL2651 light sensor, a Maxim MAX1363 $\mathrm{A} / \mathrm{D}$, and a TI TMP175 temperature sensor. The current version of the application utilizes all sensors excluding the TI temperature sensor and the Maxim A/D converter, however, due to the modularity of TinyOS programs they could be bound into the firmware in the future.

Since current off the shelf smart phones do not carry an 802.15.4 interface, a custom Bluetooth board had to be fabricated. The need for a smart phone arises from the desire to be able to transmit the gathered and processed data to a remote data store for later use. A number of various modules exist for providing serial to Bluetooth interfaces, however, Parani offers an excellent module which is small and lightweight (Parani ESD200). In addition to providing Bluetooth, the custom board breaks out a number of various interfaces offered by the PXA271, including the serial interface necessary for interfacing with the ECG module developed.

Details of the ECG module are left for Section 4.

The software running on TinyOS 2 provides data aggregation, control and some basic (for now) local processing. The current design is inspired by the well known publish/subscribe abstraction. However, due to the static nature of TinyOS applications, this breaks down into a centralized aggregation layer which handles storing and pushing the incoming data to the various processing and storage components. The data is buffered in the internal SRAM of the PXA if the Bluetooth link is not active. Local processing is currently limited to converting temperature and humidity into a bioclimatic index value [7].

\section{ECG acquisition chain}

An ECG acquisition and processing board was developed in-house and is one of the key components of this system. This particular design encompasses both an analog acquisition stage coupled with a TI MSP430 for data acquisition and signal processing. The analog stage's block diagram is shown in Figure 2. The electrode topology chosen for this design is based on a single lead with a third electrode which is switchable between ground and a right leg driven circuit. In order to reduce the effect of parasitic capacitances that appear between the cables and ground, the ground of the cable is driven by the common mode voltage signal buffered by a voltage follower. The input stage of the in-amp is based on an AC-coupled frontend [8] which provides a fully differential passive coupling network resulting in a high CMRR. A high cut off frequency low pass passive filter is used to abate RF interference. The differential signal is then amplified through a low-power, high CMRR instrumentation amplifier from Analog Devices (AD623). 


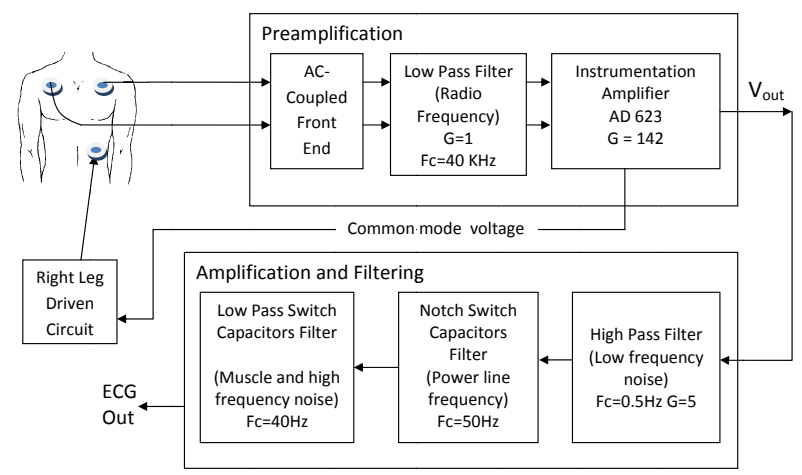

Figure 2. Analog acquisition stage block diagram

In the second stage the signal is filtered by a passive high-pass filter with a cut-off frequency of $0.5 \mathrm{~Hz}$ and further amplified in order to span the entire range of the A/D converter. Following this are 5th-order switched-capacitor filters (SCF) from Maxim; a MAX 7414 is configured to obtain a notch filter (for removing power-lines frequency); and a final MAX 7413, in its standard configuration, applies a Bessel low-pass filter at $40 \mathrm{~Hz}$. SCF are accurate, require few external components, and maintain a predictable response over the necessitated operation conditions.

Following the analog acquisition stage, a MSP430 digitizes (at $500 \mathrm{~Hz}$ ) and filters the ECG signal. The design takes advantage of the onboard ADC, hardware multiplier and utilizes one of the micro's UARTs to transmit data to the iMote2. The MSP is used to perform local processing on the ECG to extract HR and QRS wave duration. Real-time QRS detection is provided by a variant of the well known Pan and Tompkins [9] algorithm (PTA). The standard PTA flow is used, however, instead of the typical adaptive threshold stage, the algorithm uses a threshold similar to that proposed in [10].

In order to isolate the predominant QRS energy, a 17tap low-pass FIR filter with a pass-band upper frequency of $6 \mathrm{~Hz}$ and stop-band lower frequency of $30 \mathrm{~Hz}$ and then a 17-tap high-pass FIR filter with a corner frequency of 2 $\mathrm{Hz}$ was implemented. The filtered signal is then differentiated (through a 5-tap filter), squared and passed through a moving window integrator of $150 \mathrm{~ms}$.

Adaptive amplitude thresholding is finally applied to the moving integration waveform for QRS detection; two independent thresholds are used: steep slope threshold (M) and beat expectation threshold (R). A measurement algorithm calculates the QRS duration as each QRS complex is detected. Thus, two waveform features are available for subsequent arrhythmia analysis (RR interval and QRS duration).

\section{Results}

The results cover three sections: robustness, power management and finally the data output by the system.

Initial concerns in this design stemmed from on-going problems with motion artifacts and capacitive coupling through our ECG module. The first iteration of the design had problems as people walked by the person under test which would cause the output to saturate. The problem seemed to be a capacitive coupling effect between the two individuals. An AC-coupled front-end was implemented to increase the system's immunity to capacitive effects and to help balance the differential input stage [8]. Following the initial hardware design phase, the system was unable to achieve the specified behavior of the Parani ESD200 module. As the iMote 2 was powered down, the Parani module would lose its configuration. However, a solution was devised which involved placing a low-level component between the TinyOS serial stack and the UART module so that whenever the Bluetooth device was powered on or off the system would reconfigure the necessary parameters. A placement issue regarding the light sensor was noted. As a user will typically prefer to keep the iMote 2 on their belt, the angle of incidence of the ambient light will be too large to achieve an accurate and consistent reading. Some possible solutions are being considered such as creating an on body network and placing the light sensor in a more convenient location.

Power management concerns were raised with the use of Bluetooth and how the software managed its connections. Since the system has an extremely low data output rate, it seems appropriate to store some seconds worth of data and perform a burst transfer to the handheld in order to reduce the duty cycle of the Bluetooth controller. However, whenever the Parani is powered down the smart phone loses its connection. This leads to a complication in the software running on Windows Mobile. As the connection would close the software would falter. The current solution is to disable power management and power cycling in the firmware. Focus was placed on enabling appropriate power management to the MSP430. The MSP430 has two listings, one in low power mode 3 and another for when the device is active. Profiling has shown that the device stays in its low power mode for $80 \%$ of the time. This results in an average power consumption of $16.5 \mathrm{~mW}$ for the ECG module and MSP430. Table 1 lists the power consumption of the various components of the system. As seen in Table 1, managing the power on the ECG acquisition board results in a power savings of approximately $30 \%$. It is expected that extending power management to the iMote 2 and to the Bluetooth module would balance the energy consumption of the entire system, bringing the system energy requirements down into the range of $50 \mathrm{~mW}$.

After handling these and other minor issues, the sys- 
Table 1. Lists the power consumption of the different modules in the system

$\begin{array}{lccr} & \text { Current }(\mathrm{mA}) & \text { Voltage }(\mathrm{V}) & \text { Power }(\mathrm{mW}) \\ \text { Analog ECG Board } & 4.7 & 3 & 14.1 \\ \text { MSP430 LPM3 } & 0.16 & 3.3 & 0.53 \\ \text { MSP430 Active } & 3 & 3.3 & 9.9 \\ \text { iMote2 + Sensors } & 38 & 4.5 & 171 \\ \text { Bluetooth Board } & 47 & 3.28 & 154.16\end{array}$

Table 2. System Output

$\begin{array}{lccr} & \text { ID } & \text { Value } & \text { Valid } \\ \text { Accelerometer } & 0 & \text { 2d Int Array } & \text { True/False } \\ \text { Light Intensity } & 1 & 16 \text { bit Int } & \text { True/False } \\ \text { Bioclimatic Index } & 2 & 16 \text { bit Int } & \text { True/False } \\ \text { Heart Rate } & 3 & 16 \text { bit Int } & \text { True/False }\end{array}$

tem's overall goal was achieved. It is able to collect, process and transmit ECG data over Bluetooth while contextualizing the signal with bioclimatic index, light intensity and accelerometer information, see Table 2 . The valid flag is for future use, providing a mechanism to indicate if a sensor should be used, e.g. a patient removes the ECG but keeps the mote on the belt.

\section{Conclusion and future works}

This paper has presented preliminary work on an ECG platform which contextualizes raw ECG data with environmental variables as well as user activity data. A big stumbling block encountered is the wearability of this system. While each component is individually small, the system as a whole becomes cumbersome due to the ECG leads. Current research for wearable bio-systems monitoring has been geared towards the application of textile sensors. These conductive polymers woven with standard cotton and blended clothing materials allow for good contact as well as a more comfortable experience. Future designs can implement available ECG devices utilizing these textile sensors in order to make data gathering a more plausible thought.

As the issue of wearability is resolved, the next step would be to enable client-server communication. If a smart phone were to be used which integrates GPS data, then user location could be integrated into the overall data set. This would put together all the pieces necessary for a small deployment in order to gain a semantically richer data set. Further study could be performed to understand if classification algorithms would have enough data to provide appropriate event detection and help further understand the user's situation.

\section{Acknowledgements}

We appreciate the support of Intel Corporation regarding this research.

\section{References}

[1] Lee DS, Bhardwaj S, Alasaarela E, Chung WY. An ecg analysis on sensor node for reducing traffic overload in u-healthcare with wireless sensor network. Sensors 2007 IEEE 28-31 Oct. 2007;256-259. ISSN 1930-0395.

[2] Kho T, Besar R, Tan Y, Tee K, Ong K. Bluetooth-enabled ecg monitoring system. TENCON 20052005 IEEE Region 10 Nov. 2005;1-5.

[3] Healey J, Logan B. Wearable wellness monitoring using ecg and accelerometer data. Wearable Computers 2005 Proceedings Ninth IEEE International Symposium on 1821 Oct. 2005;220-221.

[4] Adler R, Flanigan M, Huang J, Kling R, Kushalnagar N, Nachman L, Wan CY, Yarvis MD. Intel mote 2: an advanced platform for demanding sensor network applications. In SenSys. 2005; 298.

[5] Ryan N. Smart Environments for Cultural Heritage. 24th International Research Symposium International Research Center for Japanese Studies ; 711 .

[6] Nachman L, Huang J, Shahabdeen J, Adler R, Kling R. Imote2: Serious computation at the edge. Wireless Communications and Mobile Computing Conference 2008 IWCMC 08 International Aug. 2008;1118-1123.

[7] Kyle W. The human bioclimate of Hong Kong. Brazil R and M Kolar Eds Proceedings of the Contemporary Climatology Conference 1994;345-350.

[8] Spinelli E, Pallas-Areny R, Mayosky M. Ac-coupled frontend for biopotential measurements. Biomedical Engineering IEEE Transactions on Mar 2003;50(3):391-395. ISSN 0018-9294.

[9] Pan J, Tompkins WJ. A real-time qrs detection algorithm. Biomedical Engineering IEEE Transactions on March 1985;BME-32(3):230-236. ISSN 0018-9294.

[10] Christov I. Real time electrocardiogram qrs detection using combined adaptive threshold. BioMedical Engineering OnLine 2004;3(1):28. ISSN 1475-925X.

Address for correspondence:

ARCES - Federico Spadini

Via Toffano, 2

Bologna, BO, 40125

Italy

fspadini@arces.unibo.it 